\title{
Valuing credit default swaps in uncertain environments
}

\author{
Yuchen $\mathrm{Li}^{1}$, Zhiqiang Zhang ${ }^{2}$, and Xiaowen Tang ${ }^{1}$ \\ ${ }^{1}$ Research Base of Beijing Modern Manufacturing Development, College of Economics and \\ Management, Beijing University of Technology, Beijing 100124, China \\ ${ }^{2}$ School of Mathematics and Computer Science, Shanxi Datong University, Datong 073009, China \\ liyuchen@bjut.edu.cn, zhangzhiqiang,zhangzqhao@126.com
}

Keywords: Uncertainty Theory, Credit Default Swap, Uncertain Finance

Abstract: The credit default swap is an important instrument in the financial market. At present, the valuation of credit default swaps is based on probability theory. In this paper, we propose a pricing formula for the credit default swap of corporate bonds from the perspective of a non-probabilistic method derived from uncertainty theory. In particular, we relate the corporate stock price to its solvency and use this relationship to develop the pricing formula. In addition, we derive two valuation models for CDS under two classic uncertain stock models. The valuation models are then compared based on their properties.

\section{Introduction}

Credit default swap (CDS) is a financial swap agreement that the seller of the CDS will compensate the buyer (usually the creditor of the reference loan) in the event of a bond default, which occurs when the bond issuer is unable to pay the full principal and interest to the investor. Therefore, if a default occurs, the bondholder with CDS receives the full amount from the CDS writer, and in return, the CDS writer receives the defaulted bond. This is similar to an insurance agreement in which a buyer (CDS buyer) protects his assets by paying a fee (CDS price) to an insurance company (CDS writer). CDS has existed since 1994, and its implementation increased in the early 2000s. Since then, CDS valuing/pricing has received a great deal of attention in academia. In this paper, we explore the valuation of CDS for corporate bonds.

\subsection{Literature Review for CDS Valuation}

The CDS valuation was first studied by Duffie (Duffie, 1999). He evaluated the CDS through a portfolio of a default-free and defaultable floating rate notes. Hull and White (Hull and White, 2000) provided a methodology for valuing CDS when the payoff was contingent on default by a single reference entity and there was no counterparty default risk. They also tested the influence of the recovery rate on the price of CDS. Later, they (Hull and White, 2001) extended their model to address CDS pricing under counterparty default risk. Researchers (Hull et al., 2004) further explored the relationship among CDS spreads, yields and credit rating announcements. Carr and Wu (Carr and Wu, 2009) proposed a dynamic framework that embodies the stock options and CDS within a single reference company. Under this framework, they proposed a tractable valuation methodology for stock options and CDS. Ammer and Cai (Ammer and Cai, 2011) investigated 
sovereign CDS pricing in nine sovereign markets. They found that CDS premiums always moved ahead of bond market movement. More recently, Brigo et al. (Brigo et al., 2014) developed an arbitrage-free valuation framework for bilateral counterparty risk and applied it to CDS valuation under collateralization. They showed that perfect collateralization cannot be achieved for CDS contracts. Bai and Wu (Bai and Wu, 2016) explained the cross-sectional variation of CDS spreads by considering the effect of firm fundamentals. The model they proposed exhibited significant explanatory power for the cross-sectional regression of market CDS. Huang et al. (Huang et al., 2017) employed stochastic differential equations (SDEs) to basket CDS pricing with contagion risk. They found sufficient conditions for the convergence of functionals associated with approximate solutions of certain path-dependent SDEs.

As is well known, most research on CDS pricing in the current literature is based on probability theory. However, it has been acknowledged that probability theory has some disadvantages in regard to valuing financial instruments. Liu (Liu, 2013) discovered a paradox that demonstrates the inappropriateness of using probability theory to describe stock prices. In the actual financial market, decision-making processes are always conducted in a state of indeterminacy, which is caused by human uncertainty and varying belief degrees. Kahneman and Tversky (Kahneman and Tversky, 1979) showed that a nonlinear transformation of the probability scale usually serves as the basis for decision making, rather than the probability itself. This finding implies that the belief degrees play an important role in real decision making. To measure the belief degree, Liu (Liu, 2007) developed uncertainty theory. Since then, it has been widely applied in many different research areas, including engineering, economics, finance, etc. Our research presents the first study of CDS valuation by means of uncertainty theory.

\subsection{Literature Review for Valuing Financial Products Using Uncertainty Theory}

Liu (Liu, 2009) introduced the uncertain differential equation and proposed a pricing formula for the European option of the uncertain stock model. The uncertain financial market is considered to be a market in which the traded financial product follows uncertain differential equations, both in theory and in application. Chen (Chen, 2011) developed an American option pricing formula for the uncertain financial market. Chen (Chen, 2013) further extended Liu's stock model by incorporating periodic dividends. He also provided a pricing formula for it. Yao and Chen (Yao and Chen, 2013) proposed a numerical method for solving uncertain differential equations and used it to calculate the European option. Zhang and Liu (Zhang and Liu, 2014) provided a valuation method for geometric Asian option pricing in the uncertain financial market. Liu et al. (Liu et al., 2015) proposed an uncertain currency model and developed a valuation method for the currency option. Yao (Yao, 2015) proved a no-arbitrage theorem for the uncertain stock model. Zhang et al. (Zhang et al., 2016) investigated the application of uncertain differential equations in power option pricing. Zhang et al. (Zhang et al., 2016) employed uncertain differential equations to price the interest rate ceiling and floor in the financial market. Zhang et al. (Zhang et al., 2017) presented a valuation method for convertible bonds under the uncertain mean-reverting stock model.

\subsection{Contributions}

Since uncertain differential equations are frequently applied to financial product valuation, we are interested in applying them to CDS pricing as well for the first time in the literature. Our contributions are as follows: 1) we consider the well-known fact that a firm's solvency is related to its stock price in CDS valuation, and 2) we provide two valuation models for CDS based on the two classic uncertain stock models.

The rest of the paper is organized as follows. In Section 2, we introduce some concepts and 
theorems from uncertainty theory. In Section 3, we introduce two pricing models for CDS based on two classical uncertain financial stock models. Examples are provided for illustrative purpose. Then, we explore the properties of the two models. Finally, Section 4 concludes the paper.

\section{Preliminaries}

Uncertain measure evaluates the personal belief degree (not frequency) of an event that may happen. Let $\Lambda$ denote an event, $M(\Lambda)$ is the belief degree that the event $\Lambda$ will occur.

Definition 2.1 Uncertainty space (Liu, 2007): let $\Gamma$ be a nonempty set, let $L$ be a $\sigma$-algebra over $\Gamma$, and let $M$ be an uncertain measure. Then the triplet $(\Gamma ; \mathrm{L} ; \mathrm{M})$ is called an uncertainty space.

Uncertain measure satisfies 4 basic axioms, which are normality, duality, subadditivity and product axioms.

Axiom 1 (Normality): let $\Gamma$ be a universal set, $M(\Gamma)=1$.

Axiom 2 (Duality): $M(\Lambda)+M\left(\Lambda^{c}\right)=1$, where $\Lambda^{c}$ are the complementary set to $\Lambda$.

Axiom 3 (Subadditivity): for every countable sequence of events $\Lambda_{1}, \Lambda_{2}, \ldots$, we have

$$
M\left\{\bigcup_{i=1}^{\infty} \Lambda_{i}\right\} \leq \sum_{i=1}^{\infty} M\left(\Lambda_{i}\right)
$$

In addition, in order to provide the operational law, Axiom 4 defines the product uncertain measure on the product $\sigma$-algebra L.

Axiom 4 (Product)(Liu, 2009):let $\left(\Gamma_{k} ; \mathrm{L}_{k} ; \mathrm{M}_{k}\right.$ ) be an uncertainty space for $k=1,2$, ... The product measure $M$ is an uncertain measure respecting

$$
M\left\{\prod_{k=1}^{\infty} \Lambda_{k}\right\}=\underset{k=1}{\infty} M_{k}\left(\Lambda_{k}\right)
$$

Where $\Lambda_{k}$ are arbitrarily chosen events from $\mathrm{L}_{k}$ for $k=1,2, \ldots$, respectively.

Theorem 2.1Monotonicity theorem (Liu, 2007): uncertain measure $M$ is a monotone increasing set function. That is, for any events $\Lambda_{1} \subset \Lambda_{2}$, we have

$$
M\left\{\Lambda_{1}\right\} \leq M\left\{\Lambda_{2}\right\}
$$

Definition 2.2 Uncertain variable (Liu, 2007): an uncertain variable is a function $\xi$ from an uncertain space $(\Gamma ; \mathrm{L} ; \mathrm{M})$ to the set of real numbers such that $\{\xi \in B\}$ is an event for any Borel set $B$.

Definition 2.3 Independence (Liu, 2009): the uncertain variables $\xi_{1}, \xi_{2}, \ldots, \xi_{m}$ are said to be independent if

$$
M\left\{\bigcap_{i=1}^{m}\left\{\xi_{i} \in B_{i}\right\}\right\}={\underset{i=1}{m}}_{\wedge} M\left(\xi_{i} \in B_{i}\right)
$$

for any Borel sets $B_{1}, B_{2}, \ldots, B_{m}$ of real numbers.

Definition 2.4(Liu, 2007) The uncertainty distribution $\Phi$ of an uncertain variable $\xi$ is defined by

$$
\Phi(x)=M(\xi \leq x)
$$

for any real number $x$.

Definition 2.5 (Liu, 2007) An uncertain variable $\xi$ is called normal if it has a normal uncertainty distribution 


$$
\Phi(x)=\left(1+\exp \left(\frac{\pi(e-x)}{\sqrt{3} \sigma}\right)\right)^{-1}
$$

denoted by $N(e, \sigma)$ where $e$ and $\sigma$ are real numbers with $\sigma>0$.

Definition 2.6 (Liu, 2007)An uncertainty distribution $\Phi(\mathrm{x})$ is said to be regular if it is a continuous and strictly increasing function with respect to $\mathrm{x}$ at which $0<\Phi(\mathrm{x})<1$, and

$$
\lim _{x \rightarrow-\infty} \Phi(x)=0, \lim _{x \rightarrow+\infty} \Phi(x)=1
$$

Theorem 2.2(Liu, 2010) Let $\xi$ be an uncertain variable with regular uncertainty distribution $\Phi$, then the expected value of $\xi$ is

$$
E(\xi)=\int_{0}^{1} \Phi^{-1}(\alpha) d \alpha
$$

where $\Phi^{-1}$ is the inverse uncertainty distribution

Definition 2.7 (Liu, 2008) Let ( $\Gamma$; L; M) be an uncertainty space and let $T$ be a totally ordered set (e.g. time). An uncertain process is a function $X_{t}(\gamma)$ from $T \times(\Gamma ; \mathrm{L} ; \mathrm{M})$ to the set of real numbers such that $\left\{X_{t} \in B\right\}$ is an event for any Borel set $B$ at each time $t$.

Remark If $X_{t}$ is an uncertain process, then $X_{t}$ is an uncertain variable at each time $t$.

Definition 2.8 (Liu, 2009) An uncertain process $C_{t}$ is said to be a Liu process if

(i) $C_{0}=0$ and almost all sample paths are Lipschitz continuous,

(ii) $C_{t}$ has stationary and independent increments, 2

(iii) every increment $C_{s+t^{-}} C_{s}$ is a normal uncertain variable with expected value 0 and variance $t$.

Definition 2.9 (Liu, 2009)Let $X_{t}$ be an uncertain process and $C_{t}$ be a Liu process. For any partition of closed interval $[a, b]$ with $a=t_{1}<t_{2}<\cdots<t_{k+1}=b$, the mesh is defined as

$$
\Delta=\max _{1 \leq i \leq k}\left|t_{i+1}-t_{i}\right|
$$

Then the Liu integral of $X_{t}$ is defined as

$$
\int_{a}^{b} X_{t} \mathrm{~d} C_{t}=\lim _{\Delta \rightarrow 0} \sum_{i=1}^{k} X_{t_{i}}\left(C_{t_{i+1}}-C_{t_{i}}\right)
$$

provided that the limit exists almost surely and is finite. In this case, the uncertain process $X_{t}$ is said to be Liu integrable.

Definition 2.10 (Liu, 2008) Suppose $C_{t}$ is a Liu process, and $f$ and $g$ are two functions. Then

$$
\mathrm{d} X_{t}=f\left(t, X_{t}\right) \mathrm{d} t+f\left(t, X_{t}\right) \mathrm{d} C_{t}
$$

is called an uncertain differential equation

Definition 2.11 (Yao and Chen, 2013) Let $\alpha$ be a number with $0<\alpha<1$. An uncertain differential equation

$$
\mathrm{d} X_{t}=f\left(t, X_{t}\right) \mathrm{d} t+g\left(t, X_{t}\right) \mathrm{d} C_{t}
$$

is said to have $\alpha$-path $X_{t}^{\alpha}$ if it solves the corresponding ordinary differential equation

$$
\mathrm{d} X_{t}^{\alpha}=f\left(t, X_{t}^{\alpha}\right) \mathrm{d} t+\left|g\left(t, X_{t}^{\alpha}\right)\right| \Phi^{-1}(\alpha) \mathrm{dt}
$$

where $\Phi^{-1}(\alpha)$ is the inverse standard normal uncertainty distribution, i.e., 


$$
\Phi^{-1}(\alpha)=\frac{\sqrt{3}}{\pi} \ln \frac{\alpha}{1-\alpha}
$$

Theorem 2.3(Yao and Chen, 2013) Let $X_{t}$ and $X_{t}^{\alpha}$ be the solution and $\alpha$-path of the uncertain differential equation

$$
\mathrm{d} X_{t}=f\left(t, X_{t}\right) \mathrm{d} t+g\left(t, X_{t}\right) \mathrm{d} C_{t}
$$

respectively. Then

$$
\begin{aligned}
& M\left\{X_{t} \leq X_{t}^{\alpha}, \forall t\right\}=\alpha \\
& M\left\{X_{t}>X_{t}^{\alpha}, \forall t\right\}=1-\alpha
\end{aligned}
$$

Theorem 2.4(Yao and Chen, 2013) Let $X_{t}$ and $X_{t}^{\alpha}$ be the solution and $\alpha$-path of the uncertain differential equation

$$
\mathrm{d} X_{t}=f\left(t, X_{t}\right) \mathrm{d} t+g\left(t, X_{t}\right) \mathrm{d} C_{t}
$$

respectively. Then the solution $X_{t}$ has an inverse uncertainty distribution

$$
\Psi_{t}^{-1}(\alpha)=X_{t}^{\alpha}
$$

\section{Valuation of the CDS}

In this section, we derive the pricing formula for CDS based on two classical uncertain financial models. Liu (Liu, 2009) proposed two uncertain processes for a stock and a bond. The stock and bond models follow uncertain differential equations in which the bond price Yt and stock price Xt are determined by

$$
\left\{\begin{array}{l}
\mathrm{d} Y_{t}=r Y_{t} \mathrm{~d} t \\
\mathrm{~d} X_{t}=\mu X_{t} \mathrm{~d} t+\sigma X_{t} \mathrm{~d} C_{t}
\end{array}\right.
$$

where $r$ is the riskless interest rate, $\mu$ is the log-drift, $\sigma$ is the log-diffusion, and $C_{t}$ is a Liu process. Eq. (1) is called the regular model.

Later, Peng and Yao (Peng and Yao, 2011) proposed two uncertain processes for a stock and a bond. The stock and bond models follow uncertain differential equations in which the bond price Yt and stock price Xt are determined by

$$
\left\{\begin{array}{l}
\mathrm{d} Y_{t}=r Y_{t} \mathrm{~d} t \\
\mathrm{~d} X_{t}=\left(m-a X_{t}\right) \mathrm{d} t+\sigma \mathrm{d} C_{t}
\end{array}\right.
$$

where $r$ is the riskless interest rate, $m$ and $a$ are constants greater than zero, $\sigma$ is the log-diffusion, and $C_{t}$ is a Liu process. Note that the bond price is $Y_{t}=Y_{0} \exp (r t)$, the stock price is $X_{t}=X_{0} \exp (\mu t+$ $\sigma C t$ ), the par value of the bond is $Y_{0}$, and the initial price of the stock is $X_{0}$. Eq. (2) is called the mean-reverting model.

To value the CDS for a corporate bond, we should estimate the rest value of the bond if it defaults. As we know, the company's ability to pay back the bond is related to its stock price in the financial market. This relationship was also discussed in Carr and Wu (Carr and Wu, 2009). They assumed that the company's bond would default when its stock price reached zero. In our paper, we make the following assumption regarding the money the company will pay to the investor (the rest value of the bond) if the bond defaults. 
Assumption 3.1 Let $p(t)$, which represents the company's solvency, denote the percentage of the par value $\left(Y_{0}\right)$ at time $t$ that the company can pay the investor if the bond defaults. Then, $p(t)$ can be estimated by the formula

$$
p(t)=\frac{\left(X_{t}-K\right)^{+}}{X_{t}}
$$

where $X_{t}$ is the stock price of the company at $t$ and $K$ is the threshold.

We assume that the company's solvency $\mathrm{p}(\mathrm{t})$ is related to the difference between the company's stock price and some threshold $\mathrm{K}$. When the stock price is lower than $\mathrm{K}$, (Xt- K)+ is equal to 0 and the investor will get nothing if the bond defaults. When the stock price is significantly larger than $\mathrm{K}$, the investor can claim almost the full value of the bond as it defaults. In a sense, when Xt is large, it is less likely that the bond will default, and even if it does, the investor can claim a large share of the par value from the company. It should be noted that $\mathrm{K}$ can be estimated by domain experts in the stock market. This simple assumption can be supported by real evidence from the financial market. For instance, Arch Coal is an American coal mining and processing company. In 2015, its miner bond defaulted, and the amount of money the bondholders received was virtually nil. In the meantime, its stock price hit bottom (traded at approximately 1 dollar per share), and the company was finally delisted from the stock market in 2016.

Next, we will derive the formula for the bondholder's loss if the bond defaults. Let us say that the bond defaults at time $t$ and the holder can recover a value of $Y 0 p(t)$. On the other hand, the holder can sell the bond for the price $Y t=Y 0 \exp (r t)$ if the bond does not default at time $t$. Here, $r$ is the riskless interest rate, which indicates the interest the bond holder will receive if the bond does not default. Hence, the loss for the holder when the bond defaults is $\mathrm{L}(\mathrm{t})=\mathrm{Y} 0 \exp (\mathrm{rt})-\mathrm{Y} 0 \mathrm{p}(\mathrm{t})$. Under the no-arbitrage assumption, the CDS price will be equal to the loss so that the holder is perfectly protected against the default risk. As we know, once CDS is purchased, the holder will not bear the default risk until the CDS expires at time T. Therefore, the value of the CDS is equal to the largest loss throughout the entire life of the CDS, from time 0 to time T. With that being said, we can find the present value of the CDS (V) by discounting back the $\mathrm{L}(\mathrm{t})$ to time 0 .

$$
\begin{aligned}
V & =\sup _{0 \leq t \leq T}(L(t) \exp (-r t)) \\
& =\sup _{0 \leq t \leq T}\left(Y_{0}(\exp (-r t)-p(t)) \exp (-r t)\right) \\
& =\sup _{0 \leq t \leq T}\left(Y_{0}\left(1-\frac{\left(X_{t}-K\right)^{+}}{X_{t}} \exp (-r t)\right)\right) .
\end{aligned}
$$

Next, we will derive the pricing formulae for CDS under the regular model (Eq. (1)) and the mean reverting model (Eq. (2)).

\subsection{Valuation of CDS Under the Regular Stock Model}

In this section, we present the pricing formula for CDS given that the underlying assets follow Eq. (1).

Definition 3.1 Assume that a credit default swap model has threshold K, riskless interest rate $\mathrm{r}$, and expiration date T. In addition, assume that the stock price follows the regular model (Eq. (1)), and the initial price for the bond and stock are $\mathrm{X} 0$ and $\mathrm{Y} 0$ where $\mathrm{X} 0 \geq \mathrm{K}$. The CDS price $\mathrm{P}$ is defined as 


$$
P=\mathrm{E}(V)=\mathrm{E}\left(\sup _{0 \leq t \leq T}\left(Y_{0}\left(1-\frac{\left(X_{t}-K\right)^{+}}{X_{t}} \exp (-r t)\right)\right)\right)
$$

Because the present value of CDS involves the stock price $\mathrm{Xt}$, which is described by an uncertain process, we define the price for CDS as the expected value of $\mathrm{V}$ as above. Next, we provide the formula for $\mathrm{P}$.

Theorem 3.1 Assume that a CDS is valued as in Definition 3.1. Then, the formula for its price is

$$
P=\int_{0}^{1} \sup _{0 \leq t \leq T}\left(Y_{0}\left(1-\frac{\left(X_{t}^{1-\alpha}-K\right)^{+}}{X_{t}^{1-\alpha}} \exp (-r t)\right)\right) \mathrm{d} \alpha
$$

where $X_{t}^{1-\alpha}=X_{0} \exp \left(\mu t+\frac{\sqrt{3} \sigma t}{\pi} \ln \left(\frac{1-\alpha}{\alpha}\right)\right)$.

\section{Proof:}

We first prove that the uncertain variable $\sup _{0 \leq t \leq T}\left(Y_{0}\left(1-\frac{\left(X_{t}-K\right)^{+}}{X_{t}} \exp (-r t)\right)\right)$ follows the inverse uncertainty distribution $\sup _{0 \leq t \leq T}\left(Y_{0}\left(1-\frac{\left(X_{t}^{1-\alpha}-K\right)^{+}}{X_{t}^{1-\alpha}} \exp (-r t)\right)\right)$.

We define the following two events:

$$
\begin{aligned}
& A=\left\{\sup _{0 \leq s \leq t}\left(Y_{0}\left(1-\frac{\left(X_{t}-K\right)^{+}}{X_{t}} \exp (-r t)\right)\right) \leq \sup _{0 \leq s \leq t}\left(Y_{0}\left(1-\frac{\left(X_{t}^{1-\alpha}-K\right)^{+}}{X_{t}^{1-\alpha}} \exp (-r t)\right)\right)\right\}, \text { It is easy to see that } A \supset\left\{X_{t} \geq X_{t}^{1-\alpha}\right\} . \text { By Theorem } \\
& B=\left\{\sup _{0 \leq s \leq t}\left(Y_{0}\left(1-\frac{\left(X_{t}-K\right)^{+}}{X_{t}} \exp (-r t)\right)\right)>\sup _{0 \leq s t r T}\left(Y_{0}\left(1-\frac{\left(X_{t}^{1-\alpha}-K\right)^{+}}{X_{t}^{1-\alpha}} \exp (-r t)\right)\right)\right\} .
\end{aligned}
$$

2.1 and Theorem 2.3, we have

$$
M\{A\} \geq M\left\{X_{t} \geq X_{t}^{1-\alpha}, \forall t\right\}=\alpha .
$$

Similarly, we also have

$$
M\{B\} \geq M\left\{X_{t}<X_{t}^{1-\alpha}, \forall t\right\}=1-\alpha .
$$

Since $A$ and $B$ are complementary events,

$$
M\{A\}+M\{B\}=1
$$

It follows from (3)-(5) that

$$
M\{A\}=\alpha,
$$

which means that the uncertain variable $\sup _{0 \leq t \leq T}\left(Y_{0}\left(1-\frac{\left(X_{t}-K\right)^{+}}{X_{t}} \exp (-r t)\right)\right)$ has the inverse uncertainty distribution $\sup _{0 \leq t \leq T}\left(Y_{0}\left(1-\frac{\left(X_{t}^{1-\alpha}-K\right)^{+}}{X_{t}^{1-\alpha}} \exp (-r t)\right)\right)$. Then, we employ Theorem 2.2 to obtain

$$
P=\int_{0}^{1} \sup _{0 \leq t \leq T}\left(Y_{0}\left(1-\frac{\left(X_{t}^{1-\alpha}-K\right)^{+}}{X_{t}^{1-\alpha}} \exp (-r t)\right)\right) \mathrm{d} \alpha .
$$

In addition, $X_{t}^{1-\alpha}$ can be calculated using Definition 2.11: 


$$
X_{t}^{1-\alpha}=X_{0} \exp \left(\mu t+\frac{\sqrt{3} \sigma t}{\pi} \ln \left(\frac{1-\alpha}{\alpha}\right)\right)
$$

Example 3.1 Suppose that the stock price follows uncertain stock model (3.2), assuming an interest rate $r=0.08$, $\log$ drift $\mu=0.1$, log-diffusion $\sigma=0.03$, initial stock and bond price $X_{0}=100$ and $Y_{0}=50$, threshold $K=10$, and expiration time $T=2$.

It follows from Definition 3.1 that the CDS price is

$$
P=\mathrm{E}\left(\sup _{0 \leq t \leq 2}\left(50\left(1-\frac{\left(X_{t}-10\right)^{+}}{X_{t}} \exp (-0.08 t)\right)\right)\right) .
$$

By Theorem 3.1, we have

$$
P=8.88
$$

Theorem 3.2 CDS valuation under the regular stock model (Eq. (4)) has the following properties.

1) $P$ is a decreasing function of $X_{0}$;

2) $P$ is an increasing function of $Y_{0}$;

3) $P$ is a decreasing function of $\mu$;

4) $P$ is a decreasing function of $\sigma$;

5) $P$ is an increasing function of $r$;

6) $P$ is an increasing function of $K$.

\section{Proof:}

1) Since $X_{t}^{1-\alpha}=X_{0} \exp \left(\mu t+\frac{\sqrt{3} \sigma t}{\pi} \ln \left(\frac{1-\alpha}{\alpha}\right)\right), X_{t}^{1-\alpha}$ is an increasing function of $X_{0} . P$ is an increasing function $X_{t}^{1-\alpha}$, so $P$ decreases with $X_{0}$.

2) It is obvious that $P$ increases with $Y_{0}$ in Eq. (4).

3) $X_{t}^{1-\alpha}$ is an increasing function of $\mu$. $P$ is an increasing function $X_{t}^{1-\alpha}$, so $P$ decreases with $\mu$.

4) $X_{t}^{1-\alpha}$ is an increasing function of $\sigma . P$ is an increasing function $X_{t}^{1-\alpha}$, so $P$ decreases with $\sigma$.

5) It is obvious that $P$ increases with $r$ in Eq. (4).

6) It is obvious that $P$ increases with $K$ in Eq. (4).

\subsection{Valuation of CDS Under the Mean-Reverting Stock Model}

In this section, we present the pricing formula for a CDS given the underlying assets, following Eq. (2). As with the valuation method in the previous section, we will first give the definition for the price of a CDS.

Definition 3.2 Assume that a credit default swap has threshold $K$, riskless interest rate $r$, and expiration date $T$. The stock price follows the mean-reverting model (Eq. (2)), and the initial price for the bond and stock are $X_{0}$ and $Y_{0}$, where $X_{0} \geq K$. The CDS price $P$ is defined as

$$
P=\mathrm{E}(V)=\mathrm{E}\left(\sup _{0 \leq t \leq T}\left(Y_{0}\left(1-\frac{\left(X_{t}-K\right)^{+}}{X_{t}} \exp (-r t)\right)\right)\right)
$$

Because the present value of the CDS involves the stock price Xt, which is described by an uncertain process, we define the price for the CDS as the expected value of $\mathrm{V}$ as above. Now, we will provide the formula for $\mathrm{P}$.

Theorem 3.3 Assume that a CDS is valued according to Definition 3.2. Then, the formula for its price is as follows. 


$$
P=\int_{0}^{1} \sup _{0 \leq t \leq T}\left(Y_{0}\left(1-\frac{\left(X_{t}^{1-\alpha}-K\right)^{+}}{X_{t}^{1-\alpha}} \exp (-r t)\right)\right) \mathrm{d} \alpha
$$

where $X_{t}^{1-\alpha}=\frac{m+\sigma \Phi^{-1}(1-\alpha)}{a}+\left(X_{0}-\frac{m+\sigma \Phi^{-1}(1-\alpha)}{a}\right) \exp (-a t)$

Proof: the proof is similar to the proof of Theorem 3.1. We first show that the uncertain variable $\sup _{0 \leq t \leq T}\left(Y_{0}\left(1-\frac{\left(X_{t}-K\right)^{+}}{X_{t}} \exp (-r t)\right)\right) \quad$ has the inverse uncertainty distribution $\sup _{0 \leq t \leq T}\left(Y_{0}\left(1-\frac{\left(X_{t}^{1-\alpha}-K\right)^{+}}{X_{t}^{1-\alpha}} \exp (-r t)\right)\right)$. Then, we apply Theorem 2.2 to obtain

$$
P=\int_{0}^{1} \sup _{0 \leq t \leq T}\left(Y_{0}\left(1-\frac{\left(X_{t}^{1-\alpha}-K\right)^{+}}{X_{t}^{1-\alpha}} \exp (-r t)\right)\right) \mathrm{d} \alpha
$$

Additionally, $X_{t}{ }^{\alpha}$ can be calculated using Definition 2.11 as follows:

$$
\begin{gathered}
X_{t}^{1-\alpha}=\frac{m+\sigma \Phi^{-1}(1-\alpha)}{a}+\left(X_{0}-\frac{m+\sigma \Phi^{-1}(1-\alpha)}{a}\right) \exp (-a t) \\
\Phi^{-1}(1-\alpha)=\frac{\sqrt{3}}{\pi} \ln \frac{1-\alpha}{\alpha}
\end{gathered}
$$

Example 3.2 Suppose that the stock price follows the uncertain stock model (Eq. (1)). Assume that $r=0.08, m=0.3, \sigma=0.03$, and $a=0.1$, the initial stock and bond price are $X_{0}=100$ and $Y_{0}=$ 50 , the threshold is $K=10$, and the expiration time is $T=2$.

It follows from Definition 3.1 that the CDS price is

$$
P=\mathrm{E}\left(\sup _{0 \leq t \leq 2}\left(50\left(1-\frac{\left(X_{t}-10\right)^{+}}{X_{t}} \exp (-0.08 t)\right)\right)\right) .
$$

By Theorem 3.1, we have

$$
P=12.44
$$

Theorem 3.4 The CDS valuation under the regular stock model (Eq. (4)) has the following properties.

1) $P$ is a decreasing function of $X_{0}$;

2) $P$ is an increasing function of $Y_{0}$;

3) $P$ is an increasing function of $r$;

4) $P$ is an increasing function of $K$.

Proof: the proof is similar to the proof of Theorem 3.2.

\subsection{Discussion}

We will now compare the properties of the two pricing formulae. In the CDS valuation formula under the regular model, Theorem 3.2 states tells us that the pattern for $\mathrm{P}$ can be found using monotonicity. In contrast, Theorem 3.4 states that $\mathrm{P}$ does not monotonically increase with $\mathrm{m}$, a and $\sigma$, which distinguishes the CDS valuation under the mean-reverting model from the valuation under the regular model. The mean reverting term $m-a X_{t}$ depends on the price change of the reference stock as follows: 1 ) When $\mathrm{m}$ is greater than aXt, the stock price tends to increase, and $\mathrm{P}$ decreases with $\mathrm{Xt}$, so $\mathrm{P}$ tends to decrease. 2 ) When $\mathrm{m}$ is less than aXt, the stock price tends to decrease, and $\mathrm{P}$ 
decreases with $\mathrm{Xt}$, so $\mathrm{P}$ tends to increase. Therefore, the CDS valuation under the mean-reverting model suggests that the CDS price will return to the long-run mean, which is a typical pattern in the financial market. The rationale behind this phenomenon is that any price that strays from the longterm norm will eventually return.

\section{Conclusions}

In this paper, we develop a closed-form valuation method for CDS of corporate bonds from the perspective of uncertainty theory. Our contribution is twofold: we prescribe two valuation models that relate the company's solvency to its stock price based on the conditions of the financial market, and we utilize the non-probabilistic methods - uncertainty theory to value CDS in which the reference company's stock price follows an uncertain process.

The current study is limited by the fact that no actual data have been employed to test and compare the proposed models. Although our models can capture the price fluctuations in CDS, the problem of finding the parameters remains to be solved. In future work, we will design methods to find the input parameters for the models.

\section{Acknowledgements}

Our work is supported by National Natural Science Foundation of China No. 71772009

\section{References}

[1] Ammer, J., Cai, F., 2011. Sovereign CDS and bond pricing dynamics in emerging markets: Does the cheapest-todeliver option matter?. Journal of International Financial Markets, Institutions and Money. 21(3), pp. 369-387.

[2] Bai, J., Wu, L., 2016. Anchoring credit default swap spreads to firm fundamentals, Journal of Financial and Quantitative Analysis. 51(5), pp. 1521-1543.

[3] Brigo, D., Capponi, A., Pallavicini, A., 2014. Arbitrage-Free Bilateral Counterparty Risk Valuation Under Collateralization and Application to Credit Default Swaps, Mathematical Finance. 24(1), pp.125-146.

[4] Carr, P., Wu, L., 2009. Stock options and credit default swaps: A joint framework for valuation and estimation, Journal of Financial Econometrics. 8(4), pp. 409-449.

[5] Chen, X., 2010. American Option Pricing Formula for Uncertain Financial Market, International Conference on Uncertainty Theory .8(2), pp.27-32.

[6] Chen, X., Liu, Y., Ralescu, D. A., 2013. Uncertain stock model with periodic dividends, Fuzzy Optimization and Decision Making. pp. 1-13.

[7] Duffie, D., 1999. Credit swap valuation. Financial Analysts Journal. 55(1), pp. 73-87.

[8] Huang, Y. T., Song, Q., Zheng, H., 2017. Weak Convergence of Path-Dependent SDEs in Basket Credit Default Swap Pricing with Contagion Risk, SIAM Journal on Financial Mathematics. 8(1), pp. 1-27.

[9] Hull, J. C., White, A. D., 2000. Valuing credit default swaps I: No counterparty default risk, The Journal of Derivatives. 8(1), pp. 29-40.

[10] Hull, J. C., White, A. D., 2001. Valuing credit default swaps II: Modeling default correlations, The Journal of derivatives. 8(3), pp. 12-21.

[11] Hull, J., Predescu, M., White, A., 2004. The relationship between credit default swap spreads, bond yields, and credit rating announcements, Journal of Banking \& Finance. 28(11), pp. 2789-2811.

[12] Kahneman, D., Tversky, A., 1979. Prospect theory. an analysis of decision making under risk, Econometrica. 47(2), pp. 263-291.

[13] Liu, B., 2007. Uncertainty Theory, Berlin: Springer

[14] Liu, B., 2008. Fuzzy process, hybrid process and uncertain process, Journal of Uncertain Systems. 2, pp.3-16.

[15] Liu, B., 2009. Some research problems in uncertainty theory, Journal of Uncertain Systems. 3(1), pp. 3-10.

[16] Liu, B., 2010. Uncertainty theory: a branch of mathematics for modeling human uncertainty.Berlin: Springer

[17] Liu,B., 2013. Toward uncertain finance theory, Journal of Uncertainty Analysis \& Applications. 1(1), pp. 1.

[18] Liu, Y., Chen, X., Ralescu, D. A., 2015. Uncertain currency model and currency option pricing, International Journal of Intelligent Systems. 30(1), pp. 40-51.

[19] Peng, J., Yao, K., 2011.A new option pricing model for stocks in uncertainty markets, International Journal of 
Operations Research. 8(2), pp. 18-26.

[20] Yao, K., Chen, X., 2013. A numerical method for solving uncertain differential equations, Journal of Intelligent \& Fuzzy Systems. 25(3), pp.825- 832.

[21] Yao, K., 2015. A no-arbitrage theorem for uncertain stock model, Fuzzy Optimization and Decision Making. 14(2), pp. 227-242.

[22] Zhang, Z., Liu, W., 2014 Geometric average Asian option pricing for uncertain financial market, Journal of Uncertain System. 8(4), pp.317-320

[23] Zhang, Z., Liu, W., Sheng, Y., 2016. Valuation of power option for uncertain financial market, Applied Mathematics and Computation. 286, pp. 257-264.

[24] Zhang, Z., Ralescu, D. A., Liu, W., 2016. Valuation of interest rate ceiling and floor in uncertain financial market, Fuzzy Optimization and Decision Making. 15(2), pp.139-154.

[25] Zhang, Z., Liu, W., Zhang, X., 2017. Valuation of convertible bond under uncertain mean-reverting stock model, Journal of Ambient Intelligence and Humanized Computing. 8(5), pp. 641-650. 